\title{
Rosen-Zener Transition in a Nonlinear Two-Level System
}

\author{
$\mathrm{Di}-\mathrm{Fa} \mathrm{Ye}^{1,2}, \mathrm{Li}-\mathrm{Bin} \mathrm{Fu}^{1}$, and $\mathrm{Jie} \mathrm{Liu}^{1} \cdot$ * \\ 1.Institute of Applied Physics and Computational Mathematics, Beijing 100088, P. R. China \\ 2. Graduate School, China Academy of Engineering Physics, Beijing 100088, P. R. China
}

\begin{abstract}
We study Rosen-Zener transition (RZT) in a nonlinear two-level system in which the level energies depend on the occupation of the levels, representing a mean-field type of interaction between the particles. We find that the nonlinearity could affect the quantum transition dramatically. At certain nonlinearity the $100 \%$ population transfer between two levels is observed and found to be robust over a very wide range of external parameters. On the other hand, the quantum transition could be completely blocked by a strong nonlinearity. In the sudden and adiabatic limits we have derived analytical expressions for the transition probability. Numerical explorations are made for a wide range of parameters of the general case. Possible applications of our theory to Bose-Einstern Condensates (BECs) are discussed.
\end{abstract}

PACS numbers: $32.80 . \mathrm{Qk}, 42.50 . \mathrm{Vk}, 03.75 . \mathrm{Lm}$

\section{INTRODUCTION}

The Rosen-Zener model was firstly proposed to study the spin-flip of two-level (Hyperfine Zeeman energy level) atoms interacting with a rotating magnetic field by N.Rosen and C.Zener to account for the double SternGerlach experiments [1]. Different from the well-known Landau-Zener model that depicts the tunnelling dynamics between two avoided-crossing energy levels [2], in the Rosen-Zener model the energy bias between two levels is fixed and the coupling between two modes is timedependent described by a rectangular [3], Gaussian [4], exponential [5] or a hyperbolic-secant functions [1]. This model constantly attracts much attention not only because it has exact analytic solution providing a bridge to understand complicated multi-mode systems [6] but also due to its versatile applications in nonresonant charge exchange of ion-atom collision [7], laser-induced excitation [8], nuclear magnetic resonance technique and quantum computation [9], to name only a few.

In the present paper, we extend the Rosen-Zener model to nonlinear case and want to see how nonlinearity affects the quantum transition dynamics in this system. Our work is motivated by recent flourish of Bose Einstein condensates (BECs), where nonlinearity naturally arises from a mean-field treatment of the interaction between particles. In fact, nonlinear effects constantly emerge in BEC system recently, such as self-trapping 10, 11, 12, 13], superfluidity [14], instability [15] and nonlinear LandauZener tunneling [16, 17]. Extending the famous RosenZener model to nonlinear case is of great interest and worthwhile at this point.

Our paper is organized as follows. In Sec.II, we introduce the nonlinear Rosen-Zener model. In Sec.III, for the degenerate case that the energy bias between two levels is zero, our numerical calculations reveal the significant effects of nonlinearity on RZT. We then derive analytic

*Email: liu jie@iapcm.ac.cn expressions for RZT in sudden and adiabatic limits. In Sec.IV, our discussions are extended to nondegenerate case. Numerical explorations for a wide range of parameters are made. Interesting phenomena are presented and discussed. Finally, in Sec.V, possible experimental realization of our model in Bose-Einstern Condensates (BECs) are discussed.

\section{NONLINEAR ROSEN-ZENER MODEL}

The nonlinear two-mode system we consider is described by following dimensionless Schrödinger equation,

$$
i \frac{\partial}{\partial t}\left(\begin{array}{l}
a \\
b
\end{array}\right)=H(t)\left(\begin{array}{l}
a \\
b
\end{array}\right)
$$

with the Hamiltonian given by

$$
H(t)=\left[\frac{\gamma}{2}+\frac{c}{2}\left(|b|^{2}-|a|^{2}\right)\right] \hat{\sigma}_{z}+\frac{v}{2} \hat{\sigma}_{x},
$$

where $\hat{\sigma}_{x}$ and $\hat{\sigma}_{z}$ are Pauli matrices, $\gamma$ and $v$ are the energy bias and coupling strength between two modes, respectively. $c$ is the nonlinear parameter describing the inter-atomic interaction. The total probability $|a|^{2}+|b|^{2}$ is conserved and set to be 1 without losing generality.

Different from the Landau-Zener type model where coupling keeps constant and energy bias varies in time linearly 2, 16, 17], in this model, the energy bias $\gamma$ and nonlinearity $c$ are set to be constant whereas the coupling $v$ is time-dependent governed by an external pulse field of the form [18],

$$
v=\left\{\begin{array}{l}
0, t<0, t>T \\
v_{0} \sin ^{2}\left(\frac{\pi t}{T}\right), t \in[0, T]
\end{array},\right.
$$

where $\mathrm{T}$ is the scanning period of the external field.

In our following study, we assume the quantum state is prepared on one mode initially. With the external field turned on, quantum transition between different modes emerges. What we concern is the population dynamics in the presence of the external field. The transition 
probability $\Gamma$ is defined as the probability of the particle occupying the other mode after the external field pulse is over.

We start our analysis with the simplest case, i.e., both the energy bias and nonlinear parameter vanish $(\gamma=0$ and $c=0)$. In this case, the Schrödinger equation denoted by Eq.(11) is solvable analytically. Setting $(a, b)=(1,0)$ as the initial condition, we readily obtain the probability of the particle populated on the other mode as the function of time,

$$
p(t)=|b(t)|^{2}=\sin ^{2}\left(v_{0} \frac{2 \pi t-T \sin \frac{2 \pi t}{T}}{8 \pi}\right),
$$

then total transition probability is obtained by substituting $t=T$ into the above equation,

$$
\Gamma=p(T)=\sin ^{2}\left(\frac{v_{0} T}{4}\right) .
$$

The above expression demonstrates a perfect Rabilike oscillation of the transition probability versus the pulse duration or scanning period $T$ of the external field. The oscillation frequency is proportional to the maximum coupling strength $v_{0}$.

\section{NONLINEAR RZT FOR DEGENERATE CASE $(\gamma=0)$}

\section{A. General Properties}

With the emergence of nonlinearity, the transition dynamics dramatically changes. In this case, the Schödinger Eq.(1) is no longer analytically solvable [19]. We therefore exploit 4-5th Runge-Kutta algorithm to trace the quantum evolution numerically and calculate the transition probability meanwhile. In our calculation, we choose the maximum coupling strength $v_{0}$ as the energy scale, thus the weak nonlinearity and strong nonlinearity refer to $c / v_{0}<<1$ and $c / v_{0}>>1$, respectively.

Our numerical results are presented in Fig 1 where the transition probability as the function of the scanning period are plotted against the nonlinear parameters that range from weak nonlinearity to strong nonlinearity. In Fig:1(a) we see a regular oscillating pattern, agreeing with the analytic prediction of formula (5). This regular periodic pattern is destroyed with the emergence of nonlinearity. For weak nonlinear case $c / v_{0}<1$, the periodicity is lost only in short pulse regime, i.e., $0<T<50$ at $c / v_{0}=0.9$. Whereas in the regime of large scanning period, a rectangular periodic pattern revives instead. The period of the rectangular oscillation increases with the enhancement of nonlinearity. The above rectangular pattern is of particular interest in practice because it represents a $100 \%$ population transfer between two modes robustly in a wide parameter regime.

For the case of strong nonlinearity, i.e., $c / v_{0}>1$ (see Fig.1d), the quantum transition between two modes is

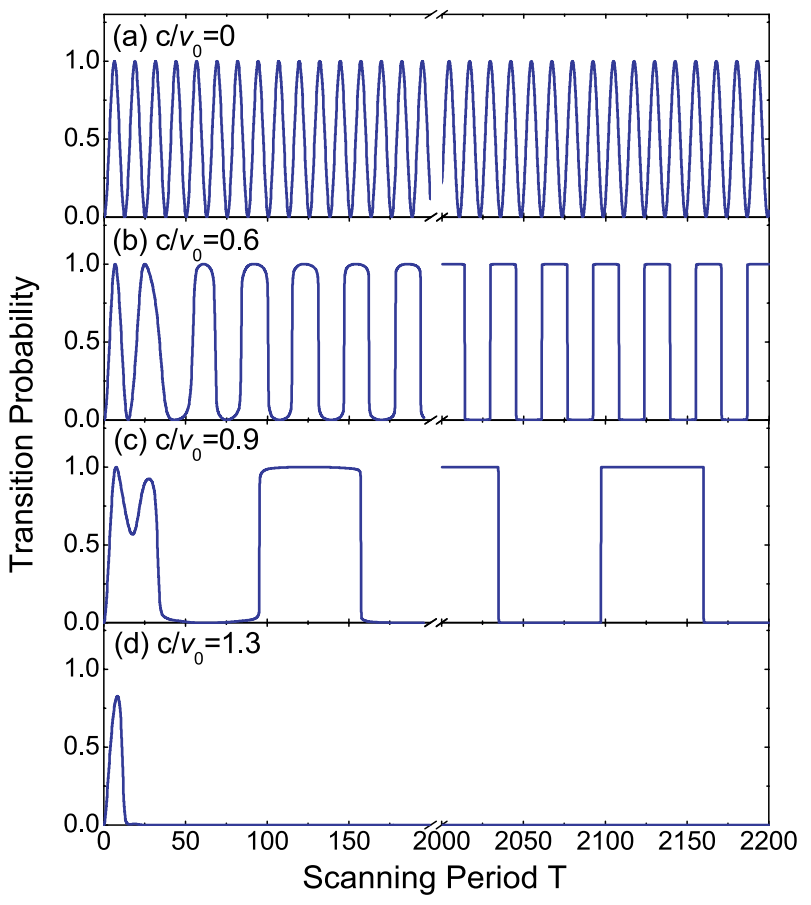

FIG. 1: (Color online.) Numerical results for the transition probability versus the scanning period under different nonlinear parameters $c / v_{0}$ (b) 0.6, (c) 0.9, (d) 1.3. For comparison, we also include the result of linear case in (a), which well reproduces the results predicted by formula (5).

even more affected by the nonlinearity: The oscillation pattern is completely broken, and when the scanning pe$\operatorname{riod} T>15$ the quantum transition is totally blocked.

The above phenomena are interesting and intriguing, need detailed investigation. Our further analysis includes two limit cases, namely, adiabatic limit and sudden limit. The adiabatic limit means that the external field varies slowly compared with the intrinsic motion of the system. From the formula(5) we see the period of intrinsic motion is characterized by $4 \pi / v_{0}$ while the external field is characterized by $T$. Thus, the adiabatic limit means $T>>4 \pi / v_{0}$ or $v_{0} T>>4 \pi$ whereas the sudden limit corresponds to $v_{0} T<<4 \pi$. In the following sections, we will further derive some analytical formulas for the transition probability and explain the above phenomena.

\section{B. Analytic Results for Sudden Limit $\operatorname{Case}\left(v_{0} T<<4 \pi\right)$}

In the sudden limit that the scanning period of the external field is small enough, it is clearly seen that nonlinearity always suppress the transition from one mode to another mode (see Fig 21). This is because of the competition between the on-site energy characterized by the nonlinear term and the hopping energy characterized by the coupling strength. For a certain coupling strength 


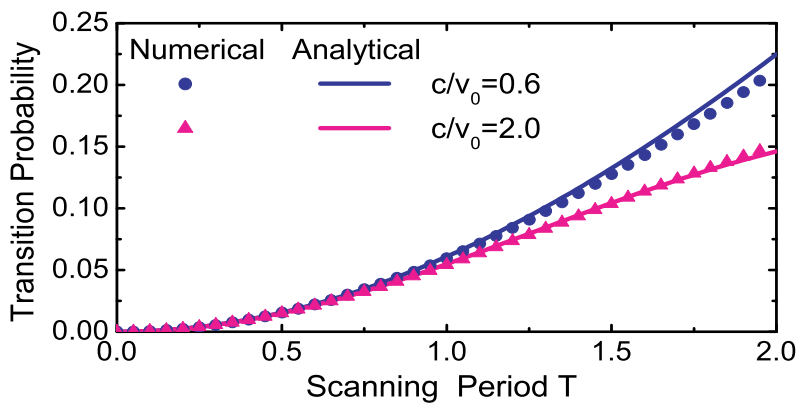

FIG. 2: (Color online). Transition probability in the sudden limit for varied nonlinear parameters. The scatters are obtained by directly integrating Eq.(1). The solid lines demonstrate the analytical results based on Eq.(9). They are in good agreement.

$v$, the larger is the nonlinear interaction $c$, the larger is the on-site energy, therefore it will block the population transfer between two modes more strongly and the oscillation between two modes becomes slower.

Now we derive some analytic results supporting the above argument. Because the transition probability is small, an explicit analytic expression can be obtained using perturbation theory. We begin with the variable transformation,

$$
\begin{gathered}
a=a^{\prime} \exp \left[-i \int_{0}^{t} \frac{c}{2}\left(\left|b^{\prime}\right|^{2}-\left|a^{\prime}\right|^{2}\right) d t\right], \\
b=b^{\prime} \exp \left[i \int_{0}^{t} \frac{c}{2}\left(\left|b^{\prime}\right|^{2}-\left|a^{\prime}\right|^{2}\right) d t\right] .
\end{gathered}
$$

As a result, the diagonal terms in the Hamiltonian are transformed away, and we have

$$
b^{\prime}(t)=\int_{0}^{t} \frac{v_{0}}{2} \sin ^{2} \frac{\pi t}{T} e^{-i \int_{0}^{t} c\left(\left|b^{\prime}\right|^{2}-\left|a^{\prime}\right|^{2}\right) d t} a^{\prime} d t .
$$

The first-order amplitude of $b^{\prime}(t)$ can be obtained by assuming $a^{\prime}=1, b^{\prime}=0$ on the right-hand side of the above equation. This yields $b^{\prime}(t)=\int_{0}^{t} \frac{v_{0}}{2} \sin ^{2} \frac{\pi t}{T} e^{i c t} d t$, and the transition probability equals to

$$
\Gamma=\frac{2 \pi^{4} v_{0}^{2}[1-\cos (c T)]}{c^{2}\left(4 \pi^{2}-c^{2} T^{2}\right)^{2}} .
$$

For linear case of $c=0$, the above formula reduces to $|b(T)|^{2}=\left(\frac{v_{0} T}{4}\right)^{2}$, which is the first order expansion of the exact solution of transition probability of Eq.(5). For the nonlinear cases, this analytical approximation is compared with our numerical results in Fig.2] and good agreement is shown. Expanding Eq.(9) using $c$ as the small parameter gives,

$$
\Gamma=\left(\frac{v_{0} T}{4}\right)^{2}\left(1-\frac{c^{2} T^{2}}{12}\right),
$$

which indicates that the quantitative decrease of transition probability is proportional to the square of nonlinear parameter $c$.
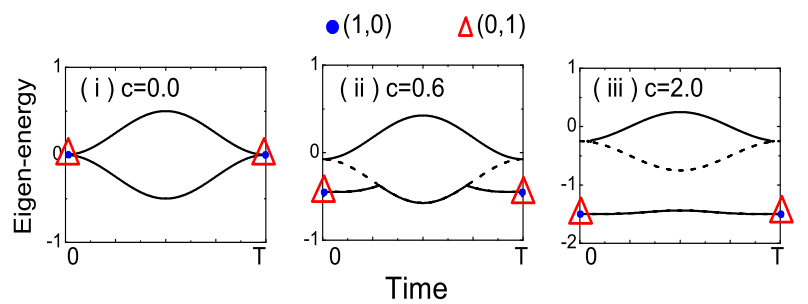

FIG. 3: Typical eigenenergy level structure in (a) linear case, (b) weak nonlinear case, (c) strong nonlinear case. The dashed lines correspond to unstable eigenstates [20].

\section{Analytic Results for Adiabatic Limit $\operatorname{Case}\left(v_{0} T>>4 \pi\right)$}

According to the adiabatic theory of nonlinear quantum mechanics 21], the characters of quantum transition in the adiabatic limit should be entirely determined by the structure of the energy levels and the properties of corresponding eigenstates. The eigenstates of the system satisfy that,

$$
\left[\frac{c}{2}\left(|b|^{2}-|a|^{2}\right) \hat{\sigma}_{z}+\frac{v}{2} \hat{\sigma}_{x}\right]\left(\begin{array}{l}
a \\
b
\end{array}\right)=\mu\left(\begin{array}{l}
a \\
b
\end{array}\right) .
$$

Solving the above nonlinear equations together with total particle conservation condition $|a|^{2}+|b|^{2}=1$, we readily obtain the chemical potential $\mu$ and the eigenstate $(a, b)$. The eigenenergies can be derived according to the relationship $\epsilon=\mu-c / 2\left(|a|^{4}+|b|^{4}\right)$. Their dependence on the parameters is plotted in Fig 3 for the cases of linearity, weak nonlinearity and strong nonlinearity, respectively. Striking phenomena are induced by the nonlinearity: Firstly, the structure of the energy levels change dramatically. In the linear case, there are two energy levels that are symmetric about a horizontal axis (see Fig.3a). However, the symmetry breaks down in the presence of nonlinearity and a new branch of eigenenergies emerges. For the weak nonlinearity (i.e., Fig.3b), at two ends and near the peak of the field pulse there exists two levels, in other regime there are three energy levels. When the nonlinearity is strong (i.e., Fig.3c), apart from the two ends, the number of the energy levels are three. Secondly, the eigenstates of the mid level( e.g. denoted by the dashed line in Fig (3) are unstable. This is evaluated by investigating the Hamiltonian-Jaccobi matrix obtained by linearizing the nonlinear equation (11) around the eigenstates. The eigenvalues of the Hamiltonian-Jaccobi matrix can be real, complex or pure imaginary. Only pure imaginary eigenvalues correspond to stable states, others indicate the unstable ones [20].

The above changes of the energy level in the presence of nonlinearity is expected to affect the quantum transition between levels greatly. However, because of the degeneracy of our concerned states of $(1,0)$ and $(0,1)$, from the above diagram of the energy levels we cannot draw a definite conclusion about the adiabatic evolution of the 


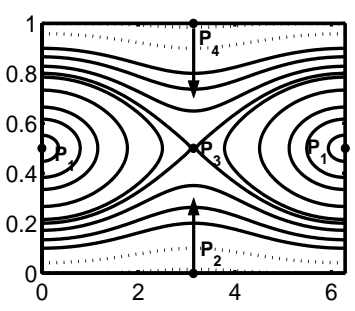

(a)
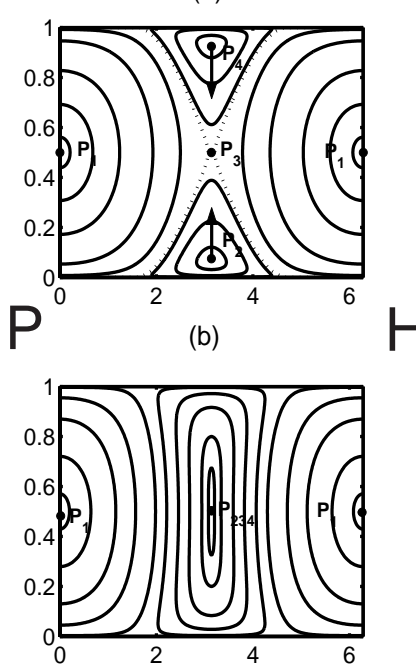

(c)

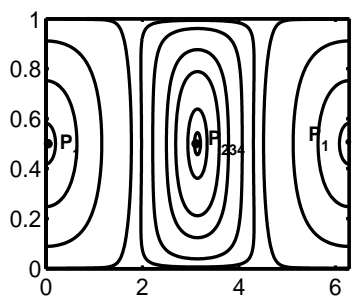

(d)

$\theta$

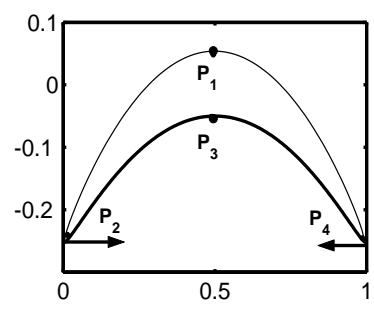

(a')
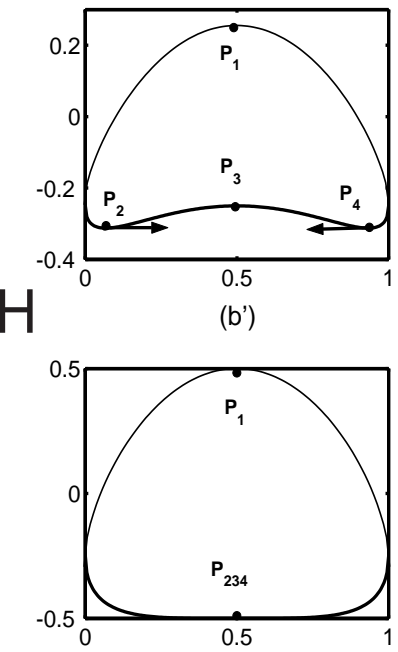

(c')

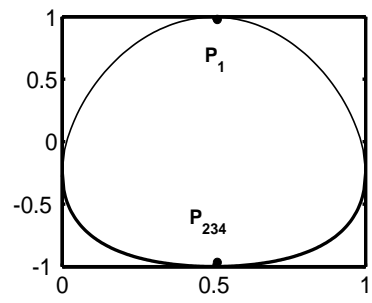

(d') $\partial H / \partial s, d s / d t=-\partial H / \partial \theta$

$$
H=v \sqrt{p(1-p)} \cos \theta-\frac{c}{4}(2 p-1)^{2} .
$$

The above classical system is capable to totally describe the dynamic properties of nonlinear quantum RosenZener system (1) on a projective Hilbert space 21]. Its fixed points, i.e., energy extrema of the classical Hamiltonian, correspond to the quantum eigenstates. For example, in Fig 4, the stable elliptic fixed point $P_{1}$ corresponds to the upper level of Fig 3 the energy of $P_{2}$ and $P_{4}$ are identical, therefore they correspond to the same energy level, i.e., the lower one in Fig 3 the saddle point $P_{3}$ is unstable, corresponding to the mid level denoted by a dashed curve in Fig 3 . The adiabatic evolution of the quantum eigenstates therefore can be evaluated by tracing the shift of the classical fixed points in the phase space when the parameter $v$ varies in time slowly [17].

The analytic expressions of the fixed points are obtained from $\dot{p}=0$ and $\dot{\theta}=0$ :

$$
\begin{gathered}
\theta^{*}=0, \pi \\
\frac{v\left(1-2 p^{*}\right)}{2 \sqrt{p^{*}\left(1-p^{*}\right)}} \cos \theta^{*}+c\left(1-2 p^{*}\right)=0 .
\end{gathered}
$$

The number of fixed points depends on the instantaneous coupling strength $v$ and the nonlinear parameter $c$. If $c / v<1$, there exists only two fixed points $\left(\theta^{*}, p^{*}\right)=$ $(0,1 / 2),(\pi, 1 / 2)$. However, when $c / v>1$, there exists four fixed points $\left(P_{1}, P_{2}, P_{3}\right.$ and $P_{4}$ in Fig $\left.4(\mathrm{a})(\mathrm{b})\right)$ : $\left(\theta^{*}, p^{*}\right)=(0,1 / 2),(\pi, 1 / 2),\left(\pi, \frac{1}{2}\left(1 \pm \sqrt{1-\frac{v^{2}}{c^{2}}}\right)\right)$. One of them $\left(P_{3}\right)$ is a saddle point, while the other three $\left(P_{1}, P_{2}\right.$ and $\left.P_{4}\right)$ are all elliptic points corresponding to the local maximum $\left(P_{1}\right)$ and minimum $\left(P_{2}\right.$ and $\left.P_{4}\right)$ of the classical Hamiltonian.

When we increase $v, P_{2}, P_{3}$ and $P_{4}$ will merge into a new stable fixed point $P_{234}$ in a regime satisfying the condition $v / c>1$, as can be seen from Fig $4(\mathrm{a} \rightarrow \mathrm{b} \rightarrow \mathrm{c} \rightarrow \mathrm{d})$. An interesting question arises when the parameter $v$ decreases to below $c$ again: Which point will the state choose to follow when $P_{234}$ bifurcates into $P_{2}, P_{3}$ and $P_{4}$ (Fig $\left.4 \mathrm{~d} \rightarrow \mathrm{c} \rightarrow \mathrm{b} \rightarrow \mathrm{a}\right)$. The state that totally follows $P_{2}$ will give a zero of the adiabatic transition probability, whereas the state that totally follows the $P_{4}$ will correspond a complete population transfer. This classical picture could explain why we see a rectangular pattern in Fig 1.

The above scenario is further supported by directly tracing the evolution of particles as shown in Fig [5] where we also demonstrate the temporal evolution of the fixed points. In the early stage, we see that the state firmly follows the fixed point $P_{2}$. It starts to show a small oscillation when the fixed points $P_{2}, P_{3}$ and $P_{4}$ merge. After that, the state either follows the fixed point $P_{2}$ or $P_{4}$. The interesting thing is that, at certain parameter, a slight pair of canonical variables of following classical Hamiltonian and satisfy the canonical equations, i.e., $d \theta / d t=$
Ref. 21] we construct the effective classical Hamiltonian with introducing two quantities: $p=|b(t)|^{2}$ as the probability of particles staying on $(0,1)$ mode, and $\theta=\theta_{a}-\theta_{b}$ as the relative phase of the two modes. They form a 


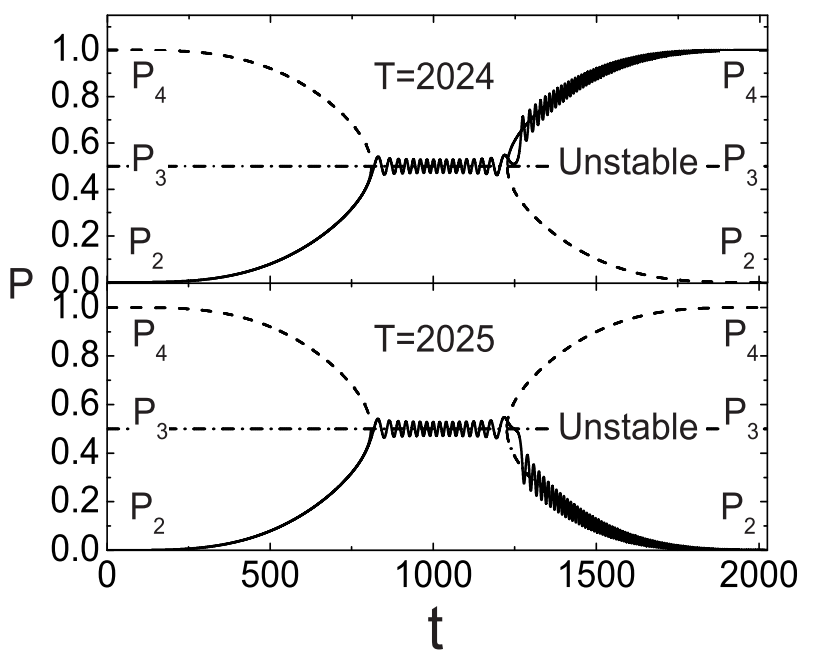

FIG. 5: Evolution of the particles and the fixed points at $c / v_{0}=0.9$. Entirely different transition probabilities are observed for a slight variation of the scanning period: (a) $\mathrm{T}=2024$, (b) $\mathrm{T}=2025$.

change on the period $\mathrm{T}$ could thoroughly change the final transition probability, a signature of the appearance of bistable state $\left(\right.$ Fig $\left.4 \mathrm{r}^{\prime} \rightarrow \mathrm{b}^{\prime}\right)$.

Our study shows that whether the state follows $P_{2}$ or $P_{4}$ is determined by the total dynamical phase accumulated during the oscillation motion around $P_{234}$, i.e. from $t^{*}$ to $t^{* *}$. Here, $t^{*}$ is the time when $P_{2}, P_{3}$ and $P_{4}$ merge into $P_{234}$, and $t^{* *}$ is the moment when $P_{234}$ bifurcates into $P_{2}, P_{3}$ and $P_{4}$. They are obtained by setting $c=v\left(t^{*}\right)=v\left(t^{* *}\right)$ :

$$
\begin{aligned}
t^{*} & =\frac{T}{\pi} \sin ^{-1} \sqrt{\frac{c}{v_{0}}} \\
t^{* *} & =T-t^{*} .
\end{aligned}
$$

To obtain the total phase, we need to calculate the instantaneous frequency that characterize the oscillations around the fixed point at first. To this end, we expand the classical Hamiltonian around the fixed point with $p=$ $1 / 2+\delta_{p}, \theta=\pi+\delta_{\theta}$, leading to

$$
\delta H=\frac{1}{4}(v-c) \delta_{p}^{2}+\frac{1}{4} v \delta_{\theta}^{2},
$$

with ignoring the higher order terms. The instantaneous frequency is then derived as

$$
\omega(t)=\frac{1}{2} \sqrt{v(t)[v(t)-c]} .
$$

Integrating $\omega(t)$ from $t^{*}$ to $t^{* *}$ gives the total phase

$$
\varphi=\int_{t^{*}}^{t^{* *}} \omega(t) d t=\frac{v_{0}-c}{4} T .
$$

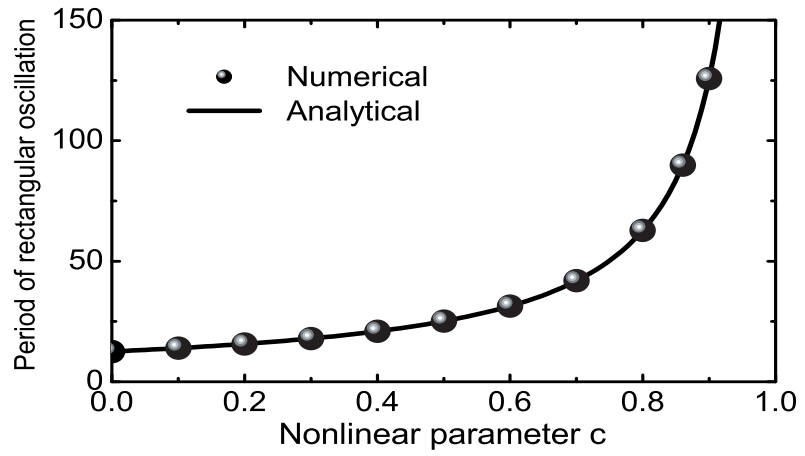

FIG. 6: Periods of rectangular oscillation as shown in Fig 1 under adiabatic limit. Numerical results are obtained by directly observing Fig 1 while the analytical curve is the plot of function $4 \pi /\left(v_{0}-c\right)$, here $v_{0}=1$. They agree well with each other.

This expression indicates that the total phase increases linearly with the scanning period. A $\pi$-value change on the phase will change the choice of the state that either follows fixed point $P_{2}$ or $P_{4}$. Thus the period of rectangular oscillation observed in Fig 1 under adiabatic limit can be expressed as

$$
T_{\text {rectangular }}=4 \pi /\left(v_{0}-c\right) .
$$

To check the above theory, we have numerically solve the nonlinear Schrödinger equation for a wide range of the parameters. The comparison between the analytical result and the numerical data shows a good agreement in Fig 6 .

The complete suppression of quantum transition under adiabatic limit in Fig प(d) can also be explained from the above picture. We briefly state it as follows. For the strong nonlinearity that $c / v_{0}>1$, the phase space evolution only undergoes Fig $4(\mathrm{a} \rightarrow \mathrm{b} \rightarrow \mathrm{a})$ as $v$ increases and decreases. During the process, no collision between the fixed points occurs. Thus the state initially populated on $P_{2}$ can safely remain on the fixed point, and finally come back to the original state smoothly. Thus no transition is observed.

\section{NONLINEAR RZT FOR NONDEGENERATE CASES $(\gamma \neq 0)$}

In this section, we extend our discussions to the nondegenerate case $(\gamma \neq 0)$. The transition probability is contour plotted with respect to the energy bias and scanning period for different nonlinear parameters ranging from weak nonlinearity to strong nonlinearity in Fig 7

The plotting reveals main features of the RZT in the nondegenerate case: i) From the first panel of Fig:7 the contour plot shows fringe structure: The bright zones correspond to high transition probability whereas dark areas indicate low transition probability. The fringe pattern is 


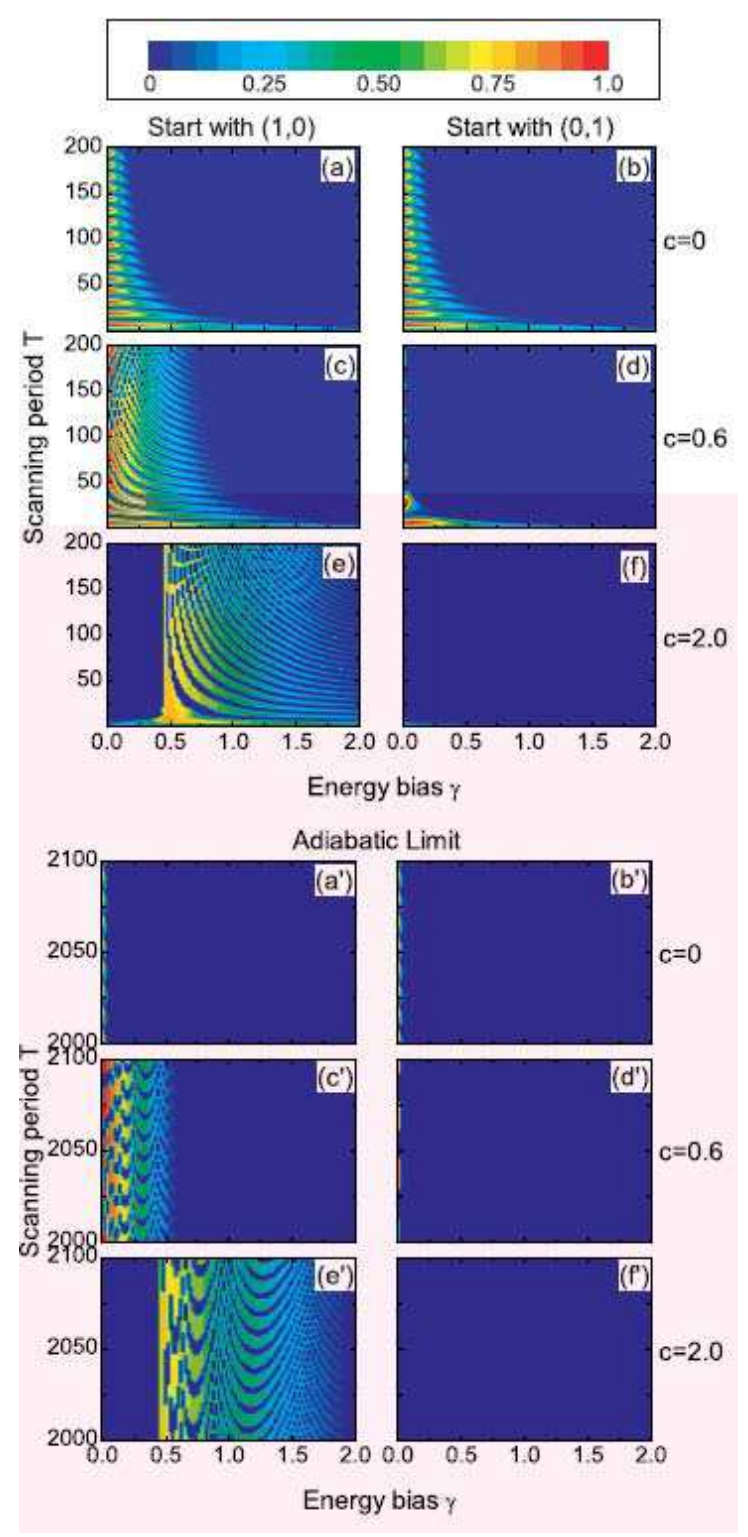

FIG. 7: (color online.) The contour plot of RZT probability as the function of energy bias and scanning period at different nonlinear parameters (upper panel) and its adiabatic counterpart (lower panel). Initially, all the particles are prepared on the high energy mode $(1,0)$ (left column) or low energy mode $(0,1)$ (right column), respectively, before the external field is turned on.

formed because of the periodic behavior of the RZT with respect to the scanning period. For linear (i.e., Fig[7 and weak nonlinear cases (i.e., Fig 7 ), this periodicity is broken by a larger energy bias; For strong nonlinear case (i.e., Fig.7 $\mathrm{f}$ ), the periodicity is absent both for larger and smaller energy bias. ii)The nonzero energy bias leads to the asymmetry of RZT plottings for the initial states of $(1,0)$ and $(0,1)$. This is clearly seen by comparing the

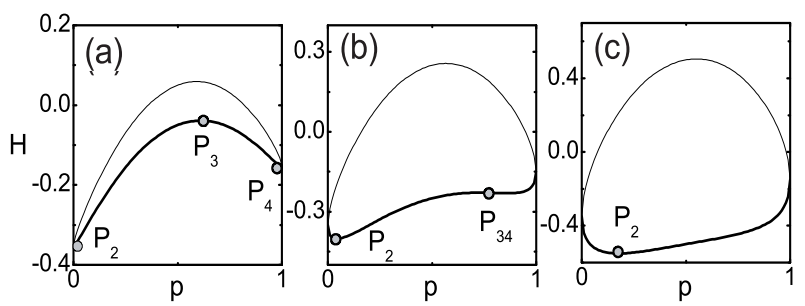

FIG. 8: Energy curve of $H$ ( Eq. (12) ) at $c=1, \gamma=0.1$, $\theta=0$ (thin line) or $\pi$ (heavy line), $v=$ (a)0.1, (b)0.5, (c)1.0. With the increase of $v, P_{3}$ and $P_{4}$ collide with each other and disappear subsequently, while $P_{2}$ maintains during the whole process.

right column with the left column of Fig.6. iii) The second panel of Fig. 6 shows the contour plot of transition probability in the adiabatic limit i.e., very large scanning period $T$. In this situation, the fringe structure is confined in a zonary region. This zonary region is of particular interest in practice because only in this region a robust population transfer between two modes is possible. Outside the zonary region, the transition probability almost equals to zero. Interestingly, the area of the zonary region broaden with increasing the nonlinear parameter $\left(\mathrm{Fig} 7 \mathrm{a}^{\prime} \rightarrow \mathrm{c}^{\prime} \rightarrow \mathrm{e}^{\prime}\right)$. When the nonlinearity is strong (i.e., Fig.6e'), the zonary region shift away from the origin. That is to say, to realize robust population transfer for the highly nonlinear case, it requires a large energy bias between two modes to compensate the difference of the on-site energy caused by the nonlinearity. iv) In the zonary region, for a fixed $\gamma$, the transition probability oscillates rectangularly versus the scanning period similar to the behavior shown in Fig.1. But the amplitude of the oscillation decreases monotonously with increasing the energy bias $\gamma$. This is indicated by the gradual darkness from left to right side in the figures. The correspondence between oscillating amplitude and energy bias suggests that the probability of the population transferred to the other mode can be designed at will by tuning the energy bias.

We can achieve insight into the above findings by analyzing the evolution of the fixed points corresponding to energy extrema. The energy curve in Fig 8 is tilted by the energy bias (for comparison one can recall Fig 《), directly leading to the asymmetry on the diagram of transition probability. The reason is stated as follows. Based on our pictures in $\operatorname{Sec} . \mathrm{III}(\mathrm{C})$, in the adiabatic limit, the transition between two modes is always accompanied by a collision between fixed points. However, with increasing $v$, the fixed point corresponding to the low energy mode $\left(P_{2}\right)$ do not collide with any other fixed points, thus there is no transition from low energy mode to higher one in the adiabatic limit. The situation is quite different for the high energy mode $\left(P_{4}\right)$, which collides with the unstable state $\left(P_{3}\right)$ and disappear subsequently as shown in Fig $[8$ (b). The condition for the occurrence of the collision 
is given by [17],

$$
v=\left(c^{\frac{2}{3}}-\gamma^{\frac{2}{3}}\right)^{\frac{3}{2}}
$$

Considering $v$ can only varies in the interval $\left[0, v_{0}\right]$, we therefore determine analytically the boundaries of the zonary regions as, $[0, c]$ if $c<v_{0}$ and $\left[\left(c^{\frac{2}{3}}-v_{0}^{\frac{2}{3}}\right)^{\frac{3}{2}}, c\right]$ if $c>v_{0}$. These two analysis is in a good agreement with the numerical results ( see Fig $8\left(c^{\prime}\right)\left(e^{\prime}\right)$ ).

Our above discussions assume that the nonlinear parameters are positive that corresponds to the repulsive interaction between particles. For the attractive interaction, the transition from $(1,0)$ mode to $(0,1)$ mode is equivalent to that transition from $(0,1)$ mode to $(1,0)$ mode for the repulsive interaction. Therefore, our discussions can be readily extended to the negative-nonlinearity case that corresponds to attractive interaction between particles. The detailed discussion is not repeated.

\section{APPLICATIONS AND DISCUSSIONS}

Our model is applicable to describe the quantum transition of two-mode BECs. One example is the BECs in a double-well. In such system, the wavefunction can be described by a superposition of two states that localize in each well separately. Then, the transition dynamics of BECs between the two wells is well described by Eq.(1) [11]. The optical double-well can be created, for example, by superimposing a blue-detuned laser beam upon the center of the magnetic trap [22]. In this case, $\gamma$ denotes the difference of the zero-point energy between two wells, $c$ is the interaction between BEC atoms that can be adjusted flexibly by Feshbach resonance, $v$ represents the height of the barrier that separate the two wells.
The barrier height can be effectively controlled by adjusting the intensity of the blue-detuned laser beam. Initially, we upload BECs atoms into one well, then ramp up and down the barrier slowly, the nonlinear Rosen-Zener transition should be observed.

Another promising candidate to observe nonlinear Rosen-Zener transition is a spinor BEC in an optical trap. In such case, a near-resonant field is applied to the condensates to couple two hyperfine states of ${ }^{87} \mathrm{Rb}$, e.g., $\left|F=1, m_{F}=-1\right\rangle$ and $\left|F=2, m_{F}=+1\right\rangle$ like in Ref. [23]. Within the standard rotating wave approximation, the system Hamiltonian can be cast into the similar form of Eq.(2) [24], where $\gamma$ denotes the frequency detuning, $c$ comes from the contributions of both the homospecies and inter-species $s$-wave scattering, $v$ is proportional to the intensity of the near-resonant laser field. After preparing the BEC atoms in one internal state, a $\sin ^{2}$ enveloped laser pulse is shined on the condensates, the nonlinear Rosen-Zener transition is expected to emerge.

In conclusion, we have investigated the Rosen-Zener transition in a nonlinear two-level system and show that the nonlinearity could dramatically affect the transition dynamics leading to many interesting phenomena. The nonlinear RZT is suggested to be observed in the twomode BEC systems.

\section{ACKNOWLEDGMENTS}

This work is supported by National Natural Science Foundation of China (No.10725521,10604009), the National Fundamental Research Programme of China under Grant No. 2006CB921400, 2007CB814800.
[1] N. Rosen and C. Zener, Phys. Rev. 40, 502 (1932).

[2] L. D. Landau, Phys. Z. Sowjetunion 2, 46 (1932); G. Zener, Proc. R. Soc. London, Ser. A 137, 696 (1932).

[3] I. I. Rabi, Phys. Rev. 51, 652 (1937).

[4] Gerald F. Thomas, Phys. Rev. A 27, 2744 (1983).

[5] V. I. Osherov and A. I. Voronin, Phys. Rev. A 49, 265 (1994).

[6] E. S. Kyoseva and N. V. Vitanov, Phys. Rev. A 73, 023420 (2006).

[7] R. E. Olson, Phys. Rev. A 6, 1822 (1972).

[8] Kalle-Antti Suominen, Barry M. Garraway, and Stig Stenholm, Phys. Rev. A 45, 3060 (1992).

[9] Michael A. Nielsen and Isaac L. Chuang, Quantum Computation and Quantum Information (Cambridge Unversity Press, Cambridge, 2000).

[10] S. Raghavan, A. Smerzi, S. Fantoni, and S. R. Shenoy, Phys. Rev. A 59, 620 (1999).

[11] Michael Albiez, Rudolf Gati, Jonas Fölling et al., Phys. Rev. Lett. 95, 010402 (2005).

[12] Guan-Fang Wang, Li-Bin Fu, and Jie Liu, Phys. Rev. A 73, 013619 (2006).
[13] T. J. Alexander, E. A. Ostrovskaya, and Y.S. Kivshar Phys. Rev. Lett. 96, 040401 (2006).

[14] Erich J. Mueller, Phys. Rev. A 66, 063603 (2002).

[15] J. R. Anglin, Phys. Rev. A 67, 051601(R) (2003); J. Liu, W. G. Wang, C. W. Zhang et al., Phys. Rev. A 72, 063623 (2005); J. Liu, C. W. Zhang, M. G. Raizen et al., Phys. Rev. A 73, 013601 (2006).

[16] Biao Wu and Qian Niu, Phys. Rev. A 61, 023402 (2000).

[17] Jie Liu, Libin Fu, Bi-Yiao Ou et al., Phys. Rev. A 66, 023404 (2002).

[18] The $\sin ^{2}$ enveloped field is usually used to simulate laser pulse. We use it to demonstrate our theory, however our main results do not sensitively depend on the shape of the pulse.

[19] Ying Wu and Xiaoxue Yang, Phys. Rev. Lett. 98, 013601 (2007).

[20] Guan-Fang Wang, Di-Fa Ye, Li-Bin Fu et al., Phys. Rev. A 74, 033414 (2006).

[21] Jie Liu, Biao Wu and Qian Niu, Phys. Rev. Lett. 90, 170404 (2003).

[22] M. R. Andrews et al., Science 275, 637 (1997). 
[23] M. R. Matthews, B. P. Anderson, P. C. Haljan et al., Phys. Rev. Lett. 83, 3358 (1999).
[24] Anthony J. Leggett, Rev. Mod. Phys. 73, 307 (2001). 